\title{
Design and Fabrication of MEMS-based Tire Pressure Sensor
}

\author{
Chia-Yen Lee ${ }^{1 *}$, Chun-Wei Yang ${ }^{1}$, Lung-Ming $\mathbf{F u}^{2}$ \\ ${ }^{1}$ Department of Vehicle Engineering, National Pingtung University of Science and Technology \\ No. 1, Shuehfu Rd., Neipu, Pingtung, Taiwan \\ leecy@mail.npust.edu.tw; stes9150133@yahoo.com.tw \\ ${ }^{2}$ Institute of Materials Engineering, National Pingtung University of Science and Technology \\ No. 1, Shuehfu Rd., Neipu, Pingtung, Taiwan \\ loudyfu@mail.npust.edu.tw
}

\section{Extended Abstract}

In recent years, emerging Micro-Electro-Mechanical Systems (MEMS) technology and micromachining techniques have been a popular approach to the miniaturization of sensors. More importantly, the functionality and reliability of these micro-sensors has been increased considerably by integrating them with mature logic IC technology or other sensors. To effectively gauge the tire pressure, it is essential to gather data of real-time tire pressure in vehicle tires. Previous studies have reported on the use of MEMS sensors for monitoring pressure parameters [1-2].

The current study developed a fabrication process utilizing Pt-piezoresistor-based pressure sensors for the identification of tire pressure. To form the tire pressure sensor, four piezoresistors were manufactured on a membrane structure released after a back-etching process. Finally, the electrical signals produced by pressure changes were amplified and converted into voltage signals using a Wheatstone bridge and an amplifier circuit (AD620) connected between the MEMS-based tire pressure sensors and an LED display.

The piezoresistors (width: $50 \mu \mathrm{m}$ ) used in the pressure sensor were deposited over silicon nitride membranes of the same dimensions $(4,000 \mu \mathrm{m} \times 4,000 \mu \mathrm{m})$, which were released by a back-etching process to form a membrane under the piezoresistorss. This was sealed with a back plate to obtain a vacuum cavity to house the pressure sensor.

Pressure measurements were carried out using an air compressor to vary the air pressure within the tire. A reference pressure meter (PG-100, Nidec Copal Electronics Corp., Japan) was used to measure the actual pressure value to calibrate the response of the sensor.

The characteristics of the tire pressure sensor were investigated as the pressure was varied between 0 and 50 psi. The measurement of pressure was carried out in a chamber connected to a tire and an air compressor to vary the pressure in the tire and the chamber. The experimental results indicated that the resistance of the pressure sensor increased linearly as the chamber pressure increased $\left(\mathrm{R}^{2}=0.978\right)$. The relationship between signal response and tire pressure illustrated in the study is given by:

$$
P=0.032 V-5.94
$$

where $P$ is the tire pressure (psi) and $V$ is the measured voltage signal $(\mathrm{mV})$. The time response $\left(\mathrm{t}_{50}\right)$ and the temperature effect of the proposed sensor are $200 \mathrm{~ms}$ and $0.0013 \mathrm{psi} /{ }^{\circ} \mathrm{C}$, respectively.

In conclusion, it is found that the voltage of the pressure sensor increased linearly as the tire pressure increased with its average sensitivity calculated as $3.2 \times 10^{-2}(\mathrm{mV} / \mathrm{psi})$ and its dimensionless sensitivity was $0.16 \mathrm{psi}^{-1}$.

\section{References}

[1] L. T. Chen, J. S. Chang, C. Y, Hsu, and W. H. Cheng, "Fabrication and performance of MEMS-based pressure sensor packages using patterned ultra-thick photoresists," Sensors, vol. 9, pp. 6200-6218, 2009.

[2] R. H. Ma, Y. H. Wang, and C. Y. Lee, "Wireless Remote Weather Monitoring System Based on MEMS Technologies," Sensors, vol. 11, pp. 2715-2727, 2011. 